PROCEEDINGS OF THE

AMERICAN MATHEMATICAL SOCIETY

Volume 125, Number 2, February 1997, Pages 523-530

S 0002-9939(97)03613-7

\title{
MEASURES WITH FINITE INDEX OF DETERMINACY OR A MATHEMATICAL MODEL FOR DR. JEKYLL AND MR. HYDE
}

\author{
CHRISTIAN BERG AND ANTONIO J. DURAN
}

(Communicated by Palle E. T. Jorgensen)

\begin{abstract}
In this note measures with finite index of determinacy (i.e. determinate measures $\mu$ for which there exists a polynomial $p$ such that $|p|^{2} \mu$ is indeterminate) are characterizated in terms of the operator associated to its Jacobi matrix. Using this characterization, we show that such determinate measures with finite index of determinacy (Jekyll) turn out to be indeterminate (Hyde) when considered as matrices of measures.
\end{abstract}

\section{Results}

By $\mathcal{M}^{*}$ we denote the set of positive measures $\mu$ on $\mathbb{R}$ having moments of every order and infinite support. A measure $\mu \in \mathcal{M}^{*}$ is determinate if no other measure has the same moments as those of $\mu$, otherwise $\mu$ is indeterminate. With $\mu \in \mathcal{M}^{*}$ we can associate the sequence $\left(p_{n}\right)_{n}$ of orthonormal polynomials. We always assume that $p_{n}$ is of degree $n$ with positive leading coefficient, and this condition together with orthonormality determines $\left(p_{n}\right)_{n}$ uniquely from $\mu$.

The sequence of polynomials $\left(p_{n}\right)_{n}$ satisfies the so-called three-term recurrence formula

$$
t p_{n}(t)=a_{n+1} p_{n+1}(t)+b_{n} p_{n}(t)+a_{n} p_{n-1}(t),
$$

where $a_{n}>0, b_{n} \in \mathbb{R}$ and $p_{-1}(t)=0$. The well-known theorem of Favard establishes that this algebraic relation characterizes the orthonormality of $\left(p_{n}\right)_{n}$ with respect to a positive measure.

The so-called Jacobi matrix is defined from the three-term recurrence relation as follows:

$$
J=\left(\begin{array}{ccccc}
b_{0} & a_{1} & 0 & 0 & 0 \\
a_{1} & b_{1} & a_{2} & 0 & 0 \\
0 & a_{2} & b_{2} & a_{3} & 0 \\
& & \ddots & \ddots & \ddots
\end{array}\right)
$$

It is the matrix representation of the operator of multiplication by $t$ in the space $\mathbb{P}$ of complex polynomials with respect to the orthonormal basis $\left(p_{n}\right)$.

Received by the editors August 29, 1995.

1991 Mathematics Subject Classification. Primary 42C05, 44A60.

This work has been partially supported by DGICYT ref. PB93-0926.

(C)1997 American Mathematical Society 
The Jacobi matrix $J$ as well as its powers $J^{k}$ define symmetric operators (possibly unbounded) in $\ell^{2}$ with domain equal to the space of sequences with only finitely many non-zero terms.

It is well-known that the deficiency index of $J$ is either $(0,0)$ or $(1,1)$ corresponding to the determinate resp. indeterminate case. We recall that a densely defined symmetric operator is called essentially self-adjoint, if its closure is self-adjoint. This is equivalent to the deficiency index being $(0,0)$.

The index of determinacy of a determinate measure $\mu$ was introduced and studied by the authors in [BD1]. This index checks the determinacy under multiplication by even powers of $|t-z|$ for $z$ a complex number, and it is defined as

$$
\operatorname{ind}_{z}(\mu)=\sup \left\{k \in \mathbb{N}|| t-\left.z\right|^{2 k} \mu \text { is determinate }\right\} .
$$

Using the index of determinacy, determinate measures can be classified as follows:

If $\mu$ is constructed from an N-extremal measure $\nu$ (i.e. $\nu$ is indeterminate and the linear space of polynomials is dense in $\left.L^{2}(\nu)\right)$ by removing the mass at $k+1$ points in the support of $\nu$, then $\mu$ is determinate with

$$
\operatorname{ind}_{z}(\mu)= \begin{cases}k, & \text { for } z \notin \operatorname{supp}(\mu), \\ k+1, & \text { for } z \in \operatorname{supp}(\mu) .\end{cases}
$$

For an arbitrary determinate measure $\mu$ the index of determinacy is either infinite for every $z$, or finite for every $z$. In the latter case the index has the form (1.1), and $\mu$ is derived from an $\mathrm{N}$-extremal measure by removing the mass at $k+1$ points.

Using that the index of determinacy is constant at complex numbers outside of the support of $\mu$, we define the index of determinacy of $\mu$ by

$$
\operatorname{ind}(\mu):=\operatorname{ind}_{z}(\mu), \quad z \notin \operatorname{supp}(\mu) .
$$

We stress that a measure $\mu$ of finite index is discrete and ind $(\mu)+1$ is the smallest degree of a polynomial $p$ such that $|p|^{2} \mu$ is indeterminate.

The purpose of this note is to prove the following characterization of a measure $\mu$ with finite index of determinacy in terms of its associated Jacobi matrix $J$ :

Theorem 1. Let $\mu$ be a determinate measure. Then the following conditions are equivalent:

(1) $\mu$ has finite index of determinacy $\operatorname{ind}(\mu)=k$.

(2) The operators $J, J^{2}, \ldots, J^{k+1}$ are essentially self-adjoint and the operator $J^{k+2}$ is not essentially self-adjoint.

(3) For any $a \in \mathbb{R}$, the operators $J+a I, J^{2}+a I, \ldots, J^{k+1}+a I$ are essentially self-adjoint and the operator $J^{k+2}+a I$ is not essentially self-adjoint.

If the conditions are fulfilled then $J^{n}+a I$ is not essentially self-adjoint for $n \geq$ $k+2$ and $a \in \mathbb{R}$.

We now show that a determinate measure with finite index of determinacy may turn out to be indeterminate when considered as a matrix of measures.

Let us put $B_{\mu}$ for the inner product defined by $\mu \in \mathcal{M}^{*}$, i.e.

$$
B_{\mu}(p, q)=\int p(t) \overline{q(t)} d \mu \text {. }
$$

This inner product has other representations using positive definite matrices of measures. Indeed, for $N \in \mathbb{N}$ we define the matrix of measures $W_{\mu}=\left(\mu_{i, j}\right)_{i, j=0}^{N-1}$ by 
$\mu_{i, j}=\psi_{N}\left(t^{i+j} \mu\right)$, the image measure under $\psi_{N}(t)=t^{N}$, i.e.

$$
\mu_{i, j}(A)=\int_{\psi_{N}^{-1}(A)} t^{i+j} d \mu(t) \quad \text { for } A \in \mathbb{B},
$$

the Borel subsets of $\mathbb{R}$. It is easy to see that $W_{\mu}$ is positive definite; i.e. for any $A \in \mathbb{B}$ the numerical matrix $W_{\mu}(A)$ is positive semidefinite. To any positive definite $N \times N$ matrix of measures $W=\left(\mu_{i, j}\right)_{i, j=0}^{N-1}$ we associate the (possibly degenerate) inner product $B_{W}$ defined by

$$
B_{W}(p, q)=\sum_{m, m^{\prime}=0}^{N-1} \int R_{N, m}(p) \overline{R_{N, m^{\prime}}(q)} d \mu_{m, m^{\prime}} \quad \text { for } p, q \in \mathbb{P},
$$

where the operators $R_{N, m}: \mathbb{P} \rightarrow \mathbb{P}, 0 \leq m \leq N-1$, are defined by

$$
R_{N, m}(p)=\sum_{n} \frac{p^{(n N+m)}(0)}{(n N+m) !} t^{n}
$$

(i.e. for every $m$, the operator $R_{N, m}$ takes from the polynomial $p$ just those powers $t^{k}$, for which $k \equiv m(\bmod N)$ and then changes $t^{n N+m}$ to $\left.t^{n}\right)$.

If $W=W_{\mu}$ with $\mu \in \mathcal{M}^{*}$ then from the definition of $R_{N, m}$, it is easy to see that

$$
\int p(t) \overline{q(t)} d \mu=\sum_{m, m^{\prime}=0}^{N-1} \int R_{N, m}(p) \overline{R_{N, m^{\prime}}(q)} d \mu_{m, m^{\prime}},
$$

i.e. $B_{\mu}=B_{W}$. (We notice that the construction of the matrix of measures $W_{\mu}$ from $\mu$ was incorrect in the introduction of [D2].)

Inner products $B_{W}$ defined from a positive definite matrix of measures $W$ as in (1.2) are characterized by the operator of multiplication by $t^{N}$ being symmetric for them, and so their sequence of orthonormal polynomials satisfies a $(2 N+1)$ term recurrence relation instead of the three-term recurrence formula satisfied by orthonormal polynomials with respect to a positive measure (see [D1] and [D2]). These inner products yield the important link between orthogonal matrix polynomials and scalar polynomials satisfying higher order recurrence relations (see [DV]).

A surprising consequence of Theorem 1 is the following:

Theorem 2. Let $\mu$ be a determinate measure with finite index of determinacy $\operatorname{ind}(\mu)=k$.

Then, for $N=1,2, \ldots, k+1$ the matrix of measures $W_{\mu}$ is the only positive definite $N \times N$ matrix of measures, such that the inner product $B_{\mu}$ is of the form (1.2). For $N \geq k+2$ there is an infinite convex set of positive definite $N \times N$ matrices of measures, such that the inner product $B_{\mu}$ is of the form (1.2).

To see this we use results linking determinacy of $N \times N$ matrices of measures and self-adjoint extensions of $J^{N}$. These results are implicitly in [D2], but for the convenience of the reader we shall state them and give complete proofs.

For each $N$ the matrix $J^{N}$ is symmetric with zeros outside the $2 N+1$ main diagonals. It is the matrix of the operator of multiplication with $t^{N}$ in $\mathbb{P}$ with respect to the orthonormal basis $\left(p_{n}\right)$ in the inner product $B_{\mu}$.

Let us consider the more general situation of an inner product $B$ on $\mathbb{P}$ with the following properties for $p, q \in \mathbb{P}$ :

(a) $B(\bar{p}, \bar{q})=\overline{B(p, q)}$

(b) $B\left(t^{N} p, q\right)=B\left(p, t^{N} q\right)$. 
We denote by $W(N)$ the convex set of positive definite $N \times N$ matrices of measures $W=\left(\mu_{i, j}\right)_{i, j=0}^{N-1}$ representing $B$ in the sense of (1.2). It is of course assumed that every polynomial is integrable with respect to every $\mu_{i, j}$. For this it is necessary and sufficient that the trace $\tau(W)=\sum \mu_{i, i}$ has moments of every order.

By (b) the operator $M: \mathbb{P} \rightarrow \mathbb{P}$ given by $M p=t^{N} p$ is symmetric in the Hilbert space $\widehat{\mathbb{P}}$, the completion of $\mathbb{P}$ with respect to $B$. Because of (a) the deficiency spaces $\Delta^{+}=\left[\left(t^{N}+i\right) \mathbb{P}\right]^{\perp}$ and $\Delta^{-}=\left[\left(t^{N}-i\right) \mathbb{P}\right]^{\perp}$ are isometric, so $M$ has equal deficiency indices. This means that $M$ has self-adjoint extensions in $\widehat{\mathbb{P}}$, and this implies, as we shall see below, that the set $W(N)$ is non-empty. The following result proves Theorem 2 .

Theorem 3. The operator $M$ is essentially self-adjoint in $\widehat{\mathbb{P}}$ if and only if $W(N)$ is a singleton.

Proof. For each self-adjoint extension $T$ of $M$ in $\widehat{\mathbb{P}}$ we consider the (projectionvalued) spectral measure $E$ of $T$ and define $W_{T}=\left(\mu_{i, j}\right)_{i, j=0}^{N-1}$ by

$$
\mu_{i, j}(A)=\left\langle E(A) t^{i}, t^{j}\right\rangle, i, j=0, \ldots, N-1, A \in \mathbb{B}(\mathbb{R}),
$$

where $\langle\cdot, \cdot\rangle$ is the inner product on $\widehat{\mathbb{P}}$ extending $B$. Then $W_{T}$ is a positive definite matrix of measures because

$$
\sum_{m, m^{\prime}=0}^{N-1} \mu_{m, m^{\prime}}(A) c_{m} \overline{c_{m^{\prime}}}=\langle E(A) p, p\rangle \geq 0
$$

with $p(t)=\sum c_{m} t^{m}$.

Since $\mathbb{P} \subset D\left(T^{n}\right)$ for all $n$, we see that each diagonal measure $\mu_{m, m}$ has moments of every order and furthermore that for any Borel set $A$

$$
\left\langle T^{n} E(A) t^{m}, t^{m^{\prime}}\right\rangle=\int_{A} s^{n} d \mu_{m, m^{\prime}}(s), m, m^{\prime}=0, \ldots, N-1 .
$$

Finally, if

$$
p(t)=\sum_{n \geq 0} \sum_{m=0}^{N-1} a_{n, m} t^{n N+m}, q(t)=\sum_{n \geq 0} \sum_{m=0}^{N-1} b_{n, m} t^{n N+m}
$$

we find using (1.5)

$$
\begin{aligned}
B(p, q) & =\sum_{n, n^{\prime} \geq 0} \sum_{m, m^{\prime}=0}^{N-1} a_{n, m} \overline{b_{n^{\prime}, m^{\prime}}} B\left(t^{n N+m}, t^{n^{\prime} N+m^{\prime}}\right) \\
& =\sum_{n, n^{\prime} \geq 0} \sum_{m, m^{\prime}=0}^{N-1} a_{n, m} \overline{b_{n^{\prime}, m^{\prime}}}\left\langle T^{n+n^{\prime}} t^{m}, t^{m^{\prime}}\right\rangle \\
& =\sum_{m, m^{\prime}=0}^{N-1} \int R_{N, m}(p) \overline{R_{N, m^{\prime}}(q)} d \mu_{m, m^{\prime}},
\end{aligned}
$$

showing that $W_{T} \in W(N)$, so in particular $W(N)$ is non-empty. 
We next claim that different self-adjoint extensions of $M$ lead to different matrices of measures. In fact, by (1.5) we have

$$
\int_{A} s^{n+n^{\prime}} d \mu_{m, m^{\prime}}(s)=\left\langle E(A) t^{n N+m}, t^{n^{\prime} N+m^{\prime}}\right\rangle,
$$

and since the polynomials $t^{n+m}, n \geq 0, m=0, \ldots, N-1$, span $\mathbb{P}$, which is dense in $\widehat{\mathbb{P}}$, it follows that the spectral measure $E$ and hence $T$ are uniquely determined from the matrix $W_{T}$.

This shows that $W(N)$ is an infinite set if $M$ is not essentially self-adjoint.

Assume finally that $M$ is essentially self-adjoint, so the closure $\bar{M}$ is the only self-adjoint extension of $M$ in $\widehat{\mathbb{P}}$. We shall show that $W_{\bar{M}}$ is the only element in $W(N)$.

Let $W=\left(\mu_{i, j}\right)$ be an arbitrary element in $W(N)$. With $W$ is associated a Hilbert space $L^{2}(W)$ of equivalence classes of $\mathbb{C}^{N}$-valued Borel functions $f=\left(f_{0}, \ldots, f_{N-1}\right)$ in the following way: all the measures $\mu_{i, j}$ in $W$ are absolutely continuous with respect to the positive trace measure $\tau(W)=\sum_{i=0}^{N-1} \mu_{i, i}$. Denoting $\phi_{i, j}$ the RadonNikodym derivative of the measure $\mu_{i, j}$ with respect to the trace measure $\tau(W)$, then $\left|\phi_{i, j}\right| \leq 1,0 \leq \phi_{i, i}$, and a vector valued function $f=\left(f_{i}\right)_{i=0}^{N-1}$ belongs to $L^{2}(W)$ if and only if the function $\sum_{i, j=0}^{N-1} f_{i} \overline{f_{j}} \phi_{i, j}$ is integrable with respect to the trace measure $\tau(W)$. The inner product is given by

$$
\langle f, f\rangle=\int \sum_{i, j=0}^{N-1} f_{i}(t) \overline{f_{j}}(t) \phi_{i, j}(t) d \tau(W)(t) .
$$

(There is a misprint in the first line of p. 96 in [D2] which has been put right in the definition of $L^{2}(W)$ given here.)

Let us consider the following operator of multiplication $T$ in $L^{2}(W)$ :

$$
D(T)=\left\{f \in L^{2}(W) \mid t f \in L^{2}(W)\right\}, T f=t f=\left(t f_{0}, \ldots, t f_{N-1}\right),
$$

which is easily seen to be self-adjoint.

The linear mapping $j: \mathbb{P} \rightarrow L^{2}(W)$ defined by

$$
j(p)=\left(R_{N, 0}(p), \ldots, R_{N, N-1}(p)\right)
$$

is an isometry, so it has a unique linear isometric extension $\hat{j}$ of $\widehat{\mathbb{P}}$ into $L^{2}(W)$. We claim that it is onto. We remark first that $\hat{j}$ is intertwining for $\bar{M}$ and $T$, i.e.

$$
\hat{j}(\bar{M}(\xi))=T(\hat{j}(\xi)) \text { for } \xi \in D(\bar{M}) .
$$

This is simply because

$$
j(M(p))=\left(R_{N, 0}\left(t^{N} p\right), \ldots, R_{N, N-1}\left(t^{N} p\right)\right)=\left(t R_{N, 0}(p), \ldots, t R_{N, N-1}(p)\right) .
$$

By the self-adjointness of $\bar{M}$ we know that $(\bar{M}-\lambda I)(D(\bar{M}))=\widehat{\mathbb{P}}$ for all $\lambda \in \mathbb{C} \backslash \mathbb{R}$. For each such $\lambda$ and $m=0, \ldots, N-1$ there exists an element $\xi_{\lambda, m} \in D(\bar{M}) \subset \widehat{\mathbb{P}}$ so that $(\bar{M}-\lambda I)\left(\xi_{\lambda, m}\right)=t^{m}$; hence by $(1.8)$

$$
(T-\lambda I)\left(\hat{j}\left(\xi_{\lambda, m}\right)\right)=\hat{j}\left((\bar{M}-\lambda I)\left(\xi_{\lambda, m}\right)\right)=j\left(t^{m}\right)=(0, \ldots, 1, \ldots, 0)
$$

with 1 at the $m$ 'th place. If we put $\hat{j}\left(\xi_{\lambda, m}\right)=\left(\varphi_{0}, \ldots, \varphi_{N-1}\right)$, we see that

$$
\varphi_{m^{\prime}}(t)=\left\{\begin{array}{l}
1 /(t-\lambda) \text { for } m^{\prime}=m \\
0 \text { for } m^{\prime} \neq m
\end{array}\right.
$$


Suppose now that $f \in L^{2}(W)$ is orthogonal to the closed subspace $\hat{j}(\widehat{\mathbb{P}})$. In particular $f \perp \hat{j}\left(\xi_{\lambda, m}\right)$; hence

$$
\int \sum_{m^{\prime}=0}^{N-1} \frac{f_{m^{\prime}}(t) \phi_{m^{\prime}, m}(t)}{t-\bar{\lambda}} d \tau(W)(t)=0,
$$

for $\lambda \in \mathbb{C} \backslash \mathbb{R}$ and $m=0, \ldots, N-1$. By the uniqueness of the Stieltjes transformation we get $\sum_{m^{\prime}=0}^{N-1} f_{m^{\prime}} \phi_{m^{\prime}, m} \tau(W)=0$ for each $m$, and in particular

$$
\int \sum_{m^{\prime}=0}^{N-1} f_{m^{\prime}} \overline{f_{m}} \phi_{m^{\prime}, m} d \tau(W)=0,
$$

which implies that

$$
\langle f, f\rangle=\int \sum_{m^{\prime}, m=0}^{N-1} f_{m^{\prime}} \overline{f_{m}} \phi_{m^{\prime}, m} d \tau(W)=0,
$$

thereby showing that $f=0$ in $L^{2}(W)$, so $\hat{j}$ is surjective. The self-adjoint operators $\bar{M}$ and $T$ are unitarily equivalent under $\hat{j}$, and in particular their spectral measures satisfy

$$
\hat{j} \circ E_{\bar{M}}(A)=E_{T}(A) \circ \hat{j}
$$

for any Borel set $A$. The spectral measure for $T$ is given as the multiplication operator $E_{T}(A) f=1_{A} f$, and we therefore have for $m, m^{\prime}=0, \ldots, N-1$

$$
\begin{aligned}
\mu_{m, m^{\prime}}(A) & =\left\langle E_{T}(A) \hat{j}\left(t^{m}\right), \hat{j}\left(t^{m^{\prime}}\right)\right\rangle=\left\langle\hat{j}\left(E_{\bar{M}}(A) t^{m}\right), \hat{j}\left(t^{m^{\prime}}\right)\right\rangle \\
& =\left\langle E_{\bar{M}}(A) t^{m}, t^{m^{\prime}}\right\rangle,
\end{aligned}
$$

which shows that $W(N)$ only consists of the matrix of measures associated with the self-adjoint extension $\bar{M}$.

Remark. When $M$ is not essentially self-adjoint, say of deficiency index $(k, k)$ with $0<k$ (and necessarily $k \leq N$ ), then the self-adjoint extensions $T$ of $M$ in $\widehat{\mathbb{P}}$ are parametrized by the unitary group $U(k)$. The corresponding matrices of measures $W_{T}$ do not fill up the whole set $W(N)$. To obtain them all one has to consider are triples $(j, H, T)$ consisting of a Hilbert space $H$, an isometric linear injection $j: \mathbb{P} \rightarrow H$ and a self-adjoint operator $T$ in $H$ with domain $D(T) \supset j(\mathbb{P})$ such that $T \circ j=j \circ M$. For such a triple the matrix $W=\left(\mu_{m, m^{\prime}}\right)$ given by

$$
\mu_{m, m^{\prime}}(A)=\left\langle E_{T}(A) j\left(t^{m}\right), j\left(t^{m^{\prime}}\right)\right\rangle
$$

belongs to $W(N)$. (Here $E_{T}$ is the spectral measure of $T$.) Any matrix of measures $W \in W(N)$ can be realized in this way by using $H=L^{2}(W), T$ and $j$ given by (1.6) and (1.7) respectively. For the matrix of measures $W_{T}$ associated with a selfadjoint extension $T$ of $M$ in $\widehat{\mathbb{P}}$ we have $\hat{j}(\mathbb{P})=L^{2}(W)$, as is easily seen by the proof in Theorem 3. On the other hand, if for $W \in W(N)$ we have that $j(\mathbb{P})$ is dense in $L^{2}(W)$, then the operator of multiplication $T$ in $L^{2}(W)$ is unitarily equivalent with a self-adjoint extension of $M$ in $\widehat{\mathbb{P}}$. 


\section{Proof of Theorem 1}

We start with the following simple observation:

Lemma. Let $\mu \in \mathcal{M}^{*}, p_{0} \in \mathbb{P}$ for which $p_{0}(t) \neq 0$ if $t \in \operatorname{supp}(\mu)$. Then $p_{0} \mathbb{P}$ is dense in $L^{2}(\mu)$ if and only if $\mathbb{P}$ is dense in $L^{2}\left(\left|p_{0}\right|^{2} \mu\right)$.

Proof. The operator $A: L^{2}\left(\left|p_{0}\right|^{2} \mu\right) \rightarrow L^{2}(\mu)$ defined by $A f=p_{0} f$ is an isometry which is onto and $A(\mathbb{P})=p_{0} \mathbb{P}$. Therefore $\mathbb{P}$ is dense in $L^{2}\left(\left|p_{0}\right|^{2} \mu\right)$ if and only if $p_{0} \mathbb{P}$ is dense in $L^{2}(\mu)$.

We now prove Theorem 1: We first prove $(1) \Leftrightarrow(2)$.

Let us consider the Hilbert space $\ell^{2}$ of complex square summable sequences and the Jacobi matrix $J$ associated to the measure $\mu$. We also let $J$ denote the operator defined by this matrix on $\ell^{2}$ with domain equal to the subspace $\mathcal{F}$ of sequences with only finitely many non-zero terms, i.e.

$$
J: \mathcal{F} \rightarrow \ell^{2}, \quad J\left(\left(a_{n}\right)_{n}\right)=\left(a_{0}, a_{1}, \ldots\right) J .
$$

Then $J$ is a symmetric operator with dense domain. As usual $\left(e_{n}\right)_{n}$ will denote the canonical basis of $\ell^{2}$.

In the space of polynomials $\mathbb{P}$, we consider the inner product $B_{\mu}$ defined by the measure $\mu$. Then the linear operator $G: \mathbb{P} \rightarrow \ell^{2}$ defined by $G\left(p_{n}\right)=e_{n}$ is an isometry from $\mathbb{P}$ endowed with the inner product $B_{\mu}$ to $\mathcal{F}$, and from the three-term recurrence relation it follows that $G^{-1} \circ J \circ G=T$ is the operator of multiplication by $t$ defined in $\mathbb{P}$. Therefore it is equivalent to prove Theorem 1 for the operator $T$ instead of $J$. Note that $\mathbb{P}$ is dense in $L^{2}(\mu)$, so $\widehat{\mathbb{P}}$ is isometrically isomorphic to $L^{2}(\mu)$.

We stress that the operator $T^{N}$ is essentially self-adjoint if and only if $\left(t^{N}+i\right) \mathbb{P}$ is dense in $L^{2}(\mu)$.

A measure $\mu$ has ind $(\mu)=k$ if and only if $\left(1+t^{2}\right)^{j} \mu$ is determinate for $j=$ $0,1, \ldots, k$ but not for $j=k+1$. Taking into account that a measure $\mu$ is determinate if and only if the polynomials are dense in $L^{2}\left(\left(1+t^{2}\right) \mu\right)$ (see $\left.[\mathrm{R}]\right)$, it follows that ind $(\mu)=k$ if and only if $\mathbb{P}$ is dense in $L^{2}\left(\left(1+t^{2}\right)^{j+1} \mu\right)$ for $j=0,1, \ldots, k$ but not for $j=k+1$. Since

$$
1 \leq \frac{\left(1+x^{2}\right)^{j}}{1+x^{2 j}} \leq 2^{j-1}, \quad j \geq 1
$$

we see that $\mathbb{P}$ is dense in $L^{2}\left(\left(1+t^{2}\right)^{j} \mu\right)$ if and only if it is dense in $L^{2}\left(\left(1+t^{2 j}\right) \mu\right)$. Therefore $\operatorname{ind}(\mu)=k$ if and only if $\mathbb{P}$ is dense in $L^{2}\left(\left(1+t^{2 j}\right) \mu\right)$ for $j=1, \ldots, k+1$ but not for $j=k+2$. From the Lemma, we get that $\operatorname{ind}(\mu)=k$ if and only if $\left(t^{j}+i\right) \mathbb{P}$ is dense in $L^{2}(\mu)$ for $j=1, \ldots, k+1$ but not for $j=k+2$, i.e. $\operatorname{ind}(\mu)=k$ if and only if $T^{j}$ is essentially self-adjoint for $j=1, \ldots, k+1$ but not for $j=k+2$.

We next prove $(2) \Leftrightarrow(3)$. For this it suffices to remark that for any $a \in \mathbb{R}$ and $j \in \mathbb{N}$, there exist two constants $C, c>0$ for which

$$
c \leq \frac{\left(a+x^{j}\right)^{2}+1}{1+x^{2 j}} \leq C \quad \text { for all } x \in \mathbb{R} .
$$

We finally note that if $\left(1+t^{2}\right)^{k+1} \mu$ is indeterminate, then $\left(1+t^{2}\right)^{n} \mu$ is indeterminate for $n \geq k+1$, and $\left(a+t^{n}+i\right) \mathbb{P}$ is not dense in $L^{2}(\mu)$ for $n \geq k+2$. 


\section{REFERENCES}

[BC] Berg, C. and Christensen, J.P.R., Density questions in the classical theory of moments, Ann. Inst. Fourier 31,3 (1981), 99-114. MR 84i:44006

[BD1] Berg, C. and Duran, A.J., The index of determinacy for measures and the $\ell^{2}$-norm of orthonormal polynomials, Trans. Amer. Math. Soc. 347 (1995), 2795-2811. MR 96f:30033

[BD2] When does a discrete differential perturbation of a sequence of orthonormal polynomials belong to $\ell^{2}$ ?, J. Funct. Anal. 136 (1996), 127-153. CMP 96:08

[D1] Duran, A.J., A generalization of Favard's theorem for polynomials satisfying a recurrence relation, J. Approx. Theory 74 (1993), 83-109. MR 94k:41008

[D2] - On Orthogonal polynomials with respect to a positive definite matrix of measures, Can. J. Math. 47 (1995), 88-112. MR 96c:42047

[DV] Duran, A.J. and van Assche, W., Orthogonal matrix polynomials and higher order recurrence relations, Linear Algebra Appl. 219 (1995), 261-280. MR 96c:42048

[R] Riesz, M., Sur le problème des moments. Troisième Note., Arkiv för Mat., astr. och fys. 17 (1923), no. 16.

Matematisk Institut, Københavns Universitet, Universitetsparken 5, DK-2100 KøbenHAVN $\varnothing$, DENMARK

E-mail address: berg@math.ku.dk

Departamento de Análisis Matemático, Universidad de Sevilla, Apdo. 1160. 41080SEvilla, Spain

E-mail address: duran@cica.es 